\title{
La liberté ou la mort. Les arguments émotionnels dans les Philippiques de Cicéron
}

Freedom or death: Emotional Arguments in Cicero's Philippics

\section{Manfred Kienpointner}

\section{OpenEdition}

Journals

Electronic version

URL: http://journals.openedition.org/aad/1786

DOI: $10.4000 /$ aad. 1786

ISSN: 1565-8961

Publisher

Université de Tel-Aviv

\section{Electronic reference}

Manfred Kienpointner, «La liberté ou la mort. Les arguments émotionnels dans les Philippiques de Cicéron », Argumentation et Analyse du Discours [Online], 13 | 2014, Online since 14 October 2014, connection on 10 December 2020. URL : http://journals.openedition.org/aad/1786 ; DOI : https:// doi.org/10.4000/aad. 1786

This text was automatically generated on 10 December 2020 .

\section{c) (†) $\ominus$}

Argumentation \& analyse du discours est mis à disposition selon les termes de la licence Creative Commons Attribution - Pas d'Utilisation Commerciale - Pas de Modification 4.0 International. 


\title{
La liberté ou la mort. Les arguments émotionnels dans les Philippiques de Cicéron
}

Freedom or death: Emotional Arguments in Cicero's Philippics

\author{
Manfred Kienpointner
}

\section{Introduction}

1 Cette étude entend explorer l'argumentation souvent très émotionnelle des Philippiques, et plus particulièrement de l'Oratio philippica secunda où Cicéron attaque son ennemi politique, Marc Antoine, avec une série d'arguments par l'émotion très agressifs (et en particulier des ad hominem). Conjointement, Cicéron utilise le concept de libertas pour donner à ses arguments plus de force émotionnelle. A la lumière des théories contemporaines de l'argumentation, on tentera ici de reconstruire et de décrire quelques-uns des arguments qui ont recours au pathos, avant de proposer une évaluation critique du discours politique cicéronien dans les Philippiques.

2 Dans ce cadre, on offrira en un premier temps une brève esquisse du traitement des arguments par l'émotion dans les théories de l'argumentation. Traditionnellement, en effet, les arguments tels que l'argumentum ad hominem, argumentum ad verecundiam, argumentum ad misericordiam, étaient classifiés comme des sophismes. Dans les théories récentes, cependant, on les traite plutôt comme des arguments sans doute faibles, mais qui n'en comportent pas moins un certain degré de plausibilité. Pour mieux juger de leur acceptabilité, on montrera qu'il convient d'analyser les arguments émotionnels dans leur contexte verbal et historique. 


\section{Les arguments émotionnels}

3 Dans le cadre du présent article, il ne peut être question de discuter le concept " émotion » de façon exhaustive. J'ai choisi de suivre la perspective cognitive sur les émotions, à savoir une position qui les caractérise essentiellement comme des jugements évaluatifs (positifs ou négatifs). Par exemple, Solomon (1993 : 209; cf. similairement Macagno et Walton $2014: 66$ ) souligne que « The heart of every emotion is its value judgments ».

4 En ce qui concerne l'histoire du traitement des « arguments en ad », rappelons que John Locke, dans son célèbre Essay Concerning Human Understanding (Locke 1824: 260 sv.), a introduit la distinction entre argumenta ad iudicium et les arguments ad verecundiam, ad hominem et ad ignorantiam désormais rangés dans la catégorie des sophismes ou paralogismes. Bien que Locke ne les désigne pas sous ces termes (Hamblin 1970 : 41), il souligne que seul un argument ad iudicium, c'est-à-dire basé sur des faits ou sur la probabilité, nous fait avancer sur la voie de la connaissance (ibid. : § 22). Après Locke, un grand nombre d'autres arguments en ad a été proposé et dénommé, entre autres les arguments ad baculum, ad misericordiam, ad odium, ad invidiam, ad metum, ad avaritiam etc. (Hamblin 1970 : 41; Kienpointner 2006). Dans les manuels de logique, ces arguments sont très souvent catégorisés comme des paralogismes («fallacies » en anglais). Par exemple, Copi (1990: 103) donne l'évaluation suivante des arguments ad populum, ad misericordiam et ad baculum :

These three fallacies, although common enough, are also so evidently fallacious as to require little explanation here. In each case the premises are plainly not relevant to the conclusion, but are deliberately chosen as instruments with which to manipulate the beliefs of the listener or reader.

5 Notons cependant que Hamblin (1970: 43) met - à juste titre - en doute la possibilité d'établir une distinction nette entre les arguments rationnels ou plausibles et les arguments fallacieux, par exemple en ce qui concerne l'argumentum ad verecundiam.

Qu'en est-il dans la théorie pragma-dialectique? La règle de pertinence («relevance rule ») semble, à première vue, y exclure tout argument émotionnel : « 4 . Standpoints may not be defended by non-argumentation or argumentation that is not relevant to the standpoint »(van Eemeren et Grootendorst 2004: 192). En fait, l'argument ad hominem est généralement une attaque personnelle qui tente de créer dans l'auditoire des émotions négatives envers l'opposant, parmi lesquelles la haine, la répulsion, le mépris. Selon cette règle, l'argument ad hominem peut être considéré comme un paralogisme (van Eemeren et Grootendorst $2004: 177)$. Mais ces auteurs (2004:2, n. 4, 195, n. 3) insistent par ailleurs sur le fait que leur caractérisation de l'argumentation comme activité rationnelle n'exclut en rien les aspects émotionnels de l'argumentation :

Of course, this does not mean that emotions have no role to play in argumentation. Not only can they be the causa of arguments, but they can also be used as arguments, rightly or wrongly.[...] rationality is a necessary condition of reasonableness, but not automatically a sufficient condition. [...] Reasonableness may sometimes even include the incorporation of emotional factors in the analysis of an argumentative discourse or text.

7 Dans la version actuelle de la pragma-dialectique, «the Extended Theory of PragmaDialectics ", les manœuvres stratégiques essayant de concilier les deux principes de la rationalité et de l'efficacité du discours argumentatif sont devenus un élément central 
de la théorie de l'argumentation (van Eemeren 2010, Kienpointner 2013). Or, parmi ces manœuvres, on trouve aussi des stratégies visant à faire plaisir à l'auditoire :

Among the starting points, for instance, that can be chosen as the point of departure in the part of the discourse that is to be reconstructed as the opening stage, arguers who maneuver strategically may be expected to try to make a selection that pleases the audience or places the case in a perspective that suits the audience (van Eemeren 2010 : 94).

8 Faut-il rappeler qu'Aristote, le fondateur de la théorie de l'argumentation européenne avec ses traités « Topiques » et « Rhétorique», soulignait encore plus clairement qu'il est légitime pour l'orateur d'utiliser non seulement des moyens persuasifs basés sur le logos (l'argumentation rationnelle), mais aussi les émotions de l'auditoire (pathos) et la réputation de l'orateur même, qui inclut notamment les émotions positives suscitées par la personnalité de l'orateur (ethos) (Arist. rhet. 1.2, 1356a 1-4). Ce fait est mis en évidence par Rubinelli :

Aristotle was enough of a realist to admit that an audience can be prompted to do something or accept certain beliefs by its emotions. Since he considers rhetoric to be the ability to find all the available means of persuasion, he includes - as the second main class of pisteis [ = moyens argumentatifs, M.K.] those relating to the audience and that appeal to their emotions (2010:53).

Plantin, qui compte parmi les plus importants spécialistes contemporains traitant de la fonction des émotions dans l'argumentation - cf. aussi Walton 1992 (traité en détail cidessous) et Gilbert (2001), pose que l'omniprésence des arguments émotionnels exige un traitement systématique, et fait la critique des théories de «l'argumentation sans émotions" (1998, 2002, 2005, 2007). En outre, il souligne qu'il y a des arguments émotionnels de qualité variable. Le caractère émotionnel n'est donc pas un critère décisif pour juger de la qualité d'un argument: «[...] de bonnes et de mauvaises argumentations peuvent susciter des émotions fortes (on peut s'enthousiasmer pour la vérité et éprouver une juste indignation); la présence d'une émotion n'est pas le critère qui permet de rejeter une argumentation » (Plantin 2005 : 102).

Enfin, Plantin attire à juste titre notre attention sur l'existence de deux dangers :

Toute approche des émotions court deux risques symétriques, l'empathie et l'alexithymie («qui n'a pas de mots pour l'émotion»). Dans la posture alexithymique, les émotions son réifiées, l'analyste les observe (ou prétend les observer) comme un astronome les galaxies. Il revendique une position non participante, coupée de l'émotion qui structure et circule autour de son objet, et se proclame libéré de tout lien, de toute empathie vis-à-vis de son objet. Le risque symétrique est précisément la " position empathique », c'est-à-dire la fusion et la confusion avec l'objet. La prétendue analyse devient une simple pseudoparticipation à l'événement [...] (2005: 104 ; cf. aussi 1998 : 9 sv.).

11 Le risque de l'empathie apparaît très clairement quand l'objet de l'analyse argumentative consiste dans les points de vue généralement approuvés ou désapprouvés par la société/communauté ambiante: les analystes risquent alors de produire une évaluation critique partiale. La même observation se retrouve chez le philosophe canadien Douglas Walton, qui réhabilite des arguments émotionnels considérés comme arguments plutôt faibles ("presumptive arguments», cf. Walton 1996: 38 sv. et Walton 1992, 1997, 1998, 1999, 2000), mais néanmoins valables pour inverser la charge de preuve dans des cas d'urgence et en l'absence d'arguments plus forts ${ }^{1}$. 

romain, et dans sa pratique discursive d'avocat et de politicien, il y eut des périodes où le courage civique et l'engagement éthique prédominaient, mais aussi des périodes où l'adaptation aux rapports de force et la poursuite de ses intérêts personnels ont été au premier plan. Ainsi, par exemple, en 80 av. J.-C., alors que Cicéron était encore un jeune avocat, il a courageusement défendu Sextus Roscius Amerinus contre l'accusation calomnieuse de Lucius Cornelius Chrysogonus, le puissant favori du dictateur Sulla. Dix ans après, Cicéron parvenait, avec ses discours In Verrem, à faire condamner Gaius Verres, qui par sa corruption sans limite s'était spectaculairement enrichi en tant que gouverneur de Sicile. En 63 av. J.-C., l'engagement énergique et les stratégies rhétoriques de Cicéron (entretemps devenu consul), dans ses discours In Catilinam ont été décisives pour forcer Catilina à quitter Rome et faire obstacle à sa conjuration et à celle de ses alliés. Publius Clodius Pulcher, Cicéron a dû s'exiler. Mais après son retour triomphant (un an plus tard), il s'est adapté de manière opportuniste à la politique de puissance du Premier Triumvirat - une alliance secrète entre César, Pompée et Crassus (60-53 av. J.-C. - cf. Fuhrmann $1992: 147$ sv.). Dans plusieurs discours, Cicéron a pris position en faveur de la politique du Triumvirat, ou a plaidé comme avocat pour des amis et alliés du Triumvirat - v. ses discours Pro Lucio Cornelio Balbo, Pro Aemilio Scauro, Pro Publio Vatinio, qualifiés de " compulsory speeches in defence of the tools of despotism » (Syme 1952: 144). Après la rupture de l'alliance entre César et Pompée (49 av. J.-C.), Cicéron n'a pris position pour Pompée contre César qu'après une très longue période d'hésitation. Pardonné par César après sa victoire, Cicéron ne voulait rien faire qui risque de lui faire 
perdre la grâce de celui-ci. Par exemple, il s'est laissé instrumentaliser par César quand il a appelé à la « clementia Caesaris » dans le discours Pro Ligario (46 av. J.-C.), où il louait le dictateur de manière (parfois ironique, mais généralement) si obséquieuse que McDermott (1970: 324, n. 1) peut caractériser le rôle de Cicéron ainsi : «Thus, a sad picture of the orator emerges, no longer king of the courts, but courting a king ».

Néanmoins, après l'assassinat de César, l'attitude et le comportement de Cicéron changent encore une fois, et ce fondamentalement (Syme 1952: 144; Stroh 2008: 107 sv.). Cicéron intervient de nouveau énergiquement dans la politique romaine et parvient, avec ses 14 Orationes Philippicae (44-43 av. J.-C.) contre le césarien Marc Antoine, à rendre "possible l'impossible ", c'est-à-dire à créer une coalition contre Marc Antoine, formée par des groupes aussi disparates que les meurtriers de César, Decimus Brutus, Marcus Brutus et Gaius Cassius, le sénat romain, et Octavien - le fils adoptif de César et futur empereur Auguste (Halfmann $2011: 82$ ). Vers la fin de l'Oratio Philippica secunda (Cic. Phil. 2.119, ainsi que Fuhrmann 1992: 291), Cicéron confirme sa volonté de risquer sa vie pour la république, si la liberté de la res publica peut être sauvée par sa mort.

18 Finalement, pourtant, Cicéron échoue et avec le Deuxième Triumvirat - Octavien, Marc Antoine et Lepidus (43 av. J.-C.) - son destin est scellé. Par ses attaques impitoyables contre Marc Antoine dans les Philippiques, il s'était créé un autre ennemi mortel. Sous la pression de celui-ci, Cicéron est donc placé sur la liste de proscription. Le sept décembre 43 av. J.-C., il est cruellement assassiné.

\subsection{Le concept cicéronien de la liberté}

Evidemment, le concept de liberté défendu par Cicéron dans ses Philippiques n'est pas à prendre dans le sens d'une démocratie moderne (Schmidt 2010). Cicéron appartenait à une société de propriétaires d'esclaves, avec une élite féodale dominante, où les femmes, même les femmes libres, n'avaient pas le droit de vote. Libertas était donc limitée à une minorité de la société romaine, les citoyens mâles libres. Dans la république tardive, même les hommes libres n'avaient pas d'influence politique s'ils n'étaient en même temps riches et de descendance noble (Bleicken $1962: 2$; Wirszubski 1967 : 48). En outre, il n'y avait qu'un suffrage censitaire (Fantham $2005: 209$ ), et le pouvoir des magistrats (en particulier des consuls) et l'influence du sénat romain ont toujours été la force dominante dans l'histoire romaine (Wirszubski 1967: 26 sv.). Le destin fatal des tribuns de la plèbe "révolutionnaires» (Titus Sempronius Gracchus, 162-133 av J.-C., et Gaius Sempronius Gracchus, 153-121 av. J.-C.) montre clairement que personne ne pouvait tenter de mettre fin à la domination du sénat sans risquer sa vie (Bleicken 1962: 3 sv.). Enfin, la liberté d'expression était limitée (Fantham 2005: 219 sv.) et, comme on sait, les riches pouvaient acheter des votes et manipuler ainsi l'opinion populaire (Wirszubski 1967 : 89).

Pour autant, cela ne signifie pas que la république romaine n'était rien de plus qu'un système dominé par l'aristocratie, ou ne se distinguait pas vraiment d'une monarchie ou d'une dictature. Elle connaissait notamment le droit de la provocatio ad populum pour protéger les simples citoyens contre l'injustice des puissants. En outre, les citoyens pouvaient aussi faire appel à l'aide des tribuns de la plèbe - iusauxilii (Fantham 2005 : 214). En particulier, le tribunus plebis constituait un contre-pouvoir considérable, avec son droit d'intercessio ( = de veto). Si Cicéron donc ne défendait pas la liberté dans un 
sens moderne, il n'en risquait pas moins sa vie pour la liberté restreinte, mais réelle d'une « démocratie quasi-féodale ».

Si on établit une comparaison entre le concept cicéronien de libertas et les concepts de liberté modernes, on trouve les différences théoriques suivantes : pour Cicéron, comme Wirszubski $(1967: 10,16)$ l'a bien montré, l'usage du lexème libertas impliquait (1) le cadre des lois romaines et (2) l'acceptation de l'inégalité sociale avec des degrés différents de dignitas (Bleicken 1962: 15). Une égalité démocratique radicale (aequabilitas) n'était donc pas acceptable pour Cicéron (Cic. rep. 1.53) ${ }^{2}$ :

(1) [E]aque quae appellatur aequabilitas, iniquissima est: cum enim par habetur honos summis et infimis, qui sint in omni populo necesse est, ipsa aequitas iniquissima est.

[D'ailleurs cette soi-disant égalité est le comble de l'iniquité ; en effet, si on accorde une égale dignité aux citoyens de la plus haute et de la moindre valeur, comme il en existe nécessairement dans n'importe quel peuple, cette égalité est la suprême iniquité].

Dans la philosophie moderne, il y a encore des points de vue relativement proches du concept de liberté défendu par Cicéron (cf. Hegel 1821/1970 : $434 \mathrm{sv.).} \mathrm{Mais} \mathrm{en} \mathrm{règle}$ générale, le concept de la liberté y est plutôt généralisé et radicalisé (cf. Kant 1788/2012; Mill 1946: 8). Pourtant, il serait injuste de blâmer Cicéron de ne pas soutenir un concept universel et radical de liberté. Il faut juger ses arguments émotionnels pour la liberté dans le contexte du concept limité de liberté qui est typique de son époque.

\section{Les arguments émotionnels de Cicéron}

Dans ce qui suit, je voudrais analyser une série d'arguments émotionnels, ou d'arguments qui comportent une dimension émotionnelle, dans l'Oratio Philippica Secunda. Pour la reconstruction explicite de l'argumentation cicéronienne, j'utilise comme outil les schèmes argumentatifs qui dévoilent non seulement la structure formelle des arguments, mais aussi les règles sémantiques ( = les topoi d'Aristote, ou les warrants de Toulmin) garantissant la pertinence des prémisses pour la conclusion. Je commence par des types d'arguments/schèmes argumentatifs qui ne sont pas intrinsèquement émotionnels, mais qui sont utilisés et formulés stratégiquement par Cicéron d'une manière (très) émotionnelle. Ces schèmes argumentatifs se trouvent (presque) tous vers la fin de la Philippica Secunda, donc à la place d'un discours (peroratio/ «péroraison ») où sont situés normalement les arguments plus émotionnels (et où l'usage des arguments émotionnels est recommandé par la rhétorique classique, cf. Cic. or. 2.232).

\subsection{L'argument d'alternatives}

À plusieurs reprises, Cicéron utilise comme technique argumentative centrale un schème qu'on pourrait appeler l'« argument d'alternatives » (Kienpointner 1993 : 2014). Toutes les alternatives d'action y sont réduites à deux - c'est « la liberté ou la mort ». La vie sans liberté, ou bien la servitude, est déclarée inacceptable, de sorte qu'il reste seulement une possibilité d'action acceptable - la mort dans la lutte pour la liberté (v. ci-dessous sur le danger de créer ainsi un faux dilemme). 

2014). Sa structure logique équivaut au syllogisme disjonctif (avec un $« \mathrm{ou} »=\mathrm{V}$ ) exclusif) :

\begin{tabular}{|ll|}
\hline Syllogisme disjonctif : & Argument d'alternatives : \\
& Ou une vie sans liberté ou la mort est préférable. \\
$\sim p$ & Une vie sans liberté n'est pas acceptable. \\
& Donc : La mort est préférable. \\
\hline
\end{tabular}

Figure 1 : Syllogisme disjonctif, argument d'alternatives

Il s'agit d'un schème argumentatif logiquement valide, c'est-à-dire que la vérité de ses prémisses entraîne nécessairement celle de sa conclusion. Il serait donc contradictoire d'affirmer les prémisses et de nier la conclusion.

Cependant, comme dans d'autres formes de l'argumentation quotidienne, la validité formelle n'est pas une garantie de l'acceptabilité sémantique (soundness) de l'argumentation. Plus spécifiquement, pour la plausibilité d'une argumentation, l'acceptabilité des prémisses ( = la vérité ou la probabilité des propositions descriptives ou la justesse des propositions normatives, selon la distinction établie par Habermas 1988 : 45) est également indispensable. Dans le cas de l'argument d'alternatives qui ne permet qu'un seule alternative, la première prémisse paraît très douteuse, parce que chaque réduction des alternatives d'action à deux alternatives uniques risque de produire le raisonnement fallacieux qu'on appelle "faux dilemme» (Perelman et Olbrechts-Tyteca $1983: 321$; Kienpointner 1993).

Et en effet, on peut généralement imaginer d'autres alternatives d'action susceptibles de résoudre aisément le dilemme postulé par la première prémisse, par exemple, des actions politiques qui atteignent les objectifs suivants: (1) une vie sans la réalisation d'une liberté politique maximale, mais au moins avec la garantie d'une liberté politique minimale ; (2) une vie sans liberté maximale, mais avec un degré de liberté politique nettement plus élevé que celui dans l'état actuel ; (3) l'émigration vers un pays avec un degré de liberté politique nettement plus élevé (etc.).

La deuxième prémisse, elle aussi, peut être mise en doute. D'un point de vue pragmatique, par exemple, on pourrait faire l'objection qu'une vie sans liberté pourrait être tolérable ou même agréable, parce qu'elle réduit la responsabilité personnelle, et la régulation stricte de la vie quotidienne vous épargne des décisions délicates et difficiles.

Etant donné les faiblesses de cette instance d'argument, on peut se demander pourquoi Cicéron l'a utilisée. La réponse à cette question réside sans doute dans l'effet émotionnel considérable du dilemme établi par la version de l'argument à deux alternatives. Car ainsi la situation est dramatisée et le pathos augmenté d'une manière très efficace. Le fait que l'on soit prêt à renoncer à la vie si cela est la seule façon de sauver la liberté de la patrie, peut toucher l'auditoire et susciter des émotions favorables à l'orateur. En outre, Cicéron construit une image valorisante de lui-même 
(ethos), c'est-à-dire l'image d'une personne courageuse, altruiste et patriotique, avec laquelle l'auditoire peut sympathiser (cf. Narducci 2009: 420, Micheli 2010: 114). Cicéron utilise le même schème argumentatif dans plusieurs passages des Philippicae Orationes (Cic. Phil. 3.29, 3.36, 6.19, 8.29) :

(2) [...] aliquando, per deos immortalis, patres conscripti, patrium animum virtutemque capiamus, ut aut libertatem propriam Romani et generis et nominis recuperemus aut mortem servituti anteponamus

[C'est pourquoi, puisque les choses en sont arrivées à ce point qu'il faut, ou bien qu'Antoine expie son crime envers la patrie, ou bien que nous soyons esclaves, par les dieux immortels! Sénateurs, reprenons enfin le courage et la vertu de nos pères, pour recouvrer la liberté, qui est le privilège de la race et du nom romains, ou préférer la mort à la servitude].

(3) Ad decus et ad libertatem nati sumus: aut haec teneamus aut cum dignitate moriamur

[Nous sommes faits pour l'honneur et pour la liberté; conservons l'un et l'autre ou mourons dans la dignité].

(4) Res in extremum est adducta discrimen; de libertate decernitur. Aut vincatis oportet, Quirites, quod profecto et pietate vestra et tanta concordia consequemini, aut quidvis potius quam serviatis. Aliae nationes servitutem pati ti possunt, populi Romani est propria libertas

[La situation est arrivée au point décisif : la lutte a pour enjeu la liberté. Il faut vaincre, Quirites, ce que vous obtiendrez assurément grâce à votre sens du devoir et à votre concorde, ou bien tout souffrir plutôt que la servitude. D'autres nations peuvent supporter la servitude, le peuple romain a le privilège de la liberté].

(5) [...] si maximum in discrimen venitur, aut libertas parata victori est aut mors proposita victo : quorum alterum optabile est, alterum effugere nemo potest. Turpis autem fuga mortis omni est morte peior

[Si la situation devient très critique, la liberté est réservée au vainqueur, la mort proposée au vaincu : l'une est souhaitable, l'autre ne peut être évitée par personne. Mais fuir honteusement la mort est pire que subir n'importe quelle mort].

31 Je reprends ici le passage décisif de l'Oratio Philippica Secunda (Cic. Phil. 2.119). Cicéron y établit le dilemme pour réaliser ses buts persuasifs (= créer des émotions favorables dans l'auditoire). Ci-dessous, je présente une reconstruction explicite de la structure de cette variante de l'argument d'alternatives, dont les parties implicites sont mises entre parenthèses :

(6) Quin etiam corpus libenter optulerim, si repraesentari morte mea libertas civitatis potest

[Bien plus : je ferai volontiers le sacrifice de ma vie, si, par ma mort, je puis réaliser pour les citoyens le rétablissement de la liberté].

[Aut] libenter moriam (= corpus libenter optulerim) si repraesentari morte mea libertas civitatis potest [aut vivam sine libertate civitatis].

Quin etiam corpus libenter optulerim si repraesentari morte mea libertas civitatis potest.

Ergo : [Non vivam sine libertas civitatis].

[Ou bien] je mourrai volontiers (= je ferai volontiers le sacrifice de ma vie), si, par ma mort, je puis réaliser pour les citoyens le rétablissement de la liberté [ou bien je vivrai sans la liberté des citoyens].

Bien plus : je ferai volontiers le sacrifice de ma vie, si, par ma mort, je puis réaliser pour les citoyens le rétablissement de la liberté.

Donc : [Je ne vivrai sans la liberté des citoyens]. 


\subsection{L'argument a minore}

Pour corroborer les prémisses douteuses de son argument d'alternatives, Cicéron lui associe d'autres schèmes argumentatifs comme l'argument a minore, déjà décrit par Aristote (top. 115a 7-8 ; rhet. 1397b 12 sv. ; cf. Perelman et Olbrechts-Tyteca 1983 : 343 , Kienpointner 1992a : 284 sv. ; Kienpointner 1992b ; Walton et al. $2008: 100$ sv.). Ce type d'argument repose sur une comparaison entre deux propositions qui décrivent deux situations avec un degré de probabilité clairement différent. Si même la proposition moins probable (a minore) est vraie, alors la proposition plus probable est encore plus vraie. La structure du schème argumentatif a minore peut être représentée comme suit (figure 2) :

\begin{tabular}{|ll|}
\hline Modus ponens : & Argument « a minore » : \\
$\mathrm{p} \rightarrow \mathrm{q}$ & Si même la proposition P est vraie, qui est moins probable que la \\
& proposition Q, Q est davantage vraie. \\
$\mathrm{p}$ & Même P est vraie. \\
& \\
\hline Donc : q. & Donc : Q est davantage vraie. \\
\end{tabular}

Figure 2 : Modus ponens, Argument « a minore »

Comme l'argument d'alternatives, l'argument a minore est logiquement valide: la structure logique est celle du schème d'inférence valide Modus ponens. Pourtant, encore une fois, on ne peut juger la plausibilité du schème argumentatif sans évaluer la vérité/ probabilité/justesse des prémisses.

Cicéron utilise deux arguments a minore, qui sont placés l'un avant, l'autre après son argument d'alternatives. Tous les deux essaient de prouver que Cicéron n'avait que deux options, mourir ou vivre sans liberté. Le premier argument a minore exclut la possibilité d'un compromis en ce qui concerne la liberté de la république romaine (cf. les options d'action 1 à 3 mentionnés ci-dessus) : Cicéron souligne que ses antécédents ne lui permettent qu'une lutte énergique pour la liberté, quel qu'en soit le prix. Déjà, jeune, il luttait contre Catilina, donc maintenant qu'il est un politicien âgé, il doit défendre encore davantage la république contre Marc Antoine (Cic. Phil. 2.118) :

(7) Defendi rem publicam adulescens, non deseram senex: contempsi Catilinae gladios, non pertimescam tuos

[J'ai défendu la République dans ma jeunesse, je ne l'abandonnerai pas dans ma vieillesse ; j'ai méprisé les épées de Catilina, je ne redouterai pas les tiennes].

Les antithèses (adulescens - senex, Catilinae gladios - tuos [sc. gladios]) et la métonymie gladios (les armes au lieu des soldats de l'ennemi Marc Antoine) soulignent dramatiquement que les deux situations historiques sont identiques quant à l'importance du danger couru par Cicéron. Seul l'âge les différencie - or, un homme âgé a moins à perdre. Encore une fois, Cicéron essaie de créer l'image d'un homme courageux, et donc une image favorable de l'orateur (ethos), en suscitant respect et admiration dans l'auditoire. 
Le deuxième argument $a$ minore de Cicéron évoque le fait que vingt ans auparavant il avait déjà contesté qu'un consulaire ne pouvait mourir trop tôt (cf. aussi les remarques pertinentes de Cicéron dans le quatrième discours contre Catilina : Catil. 4.3). Puisque Cicéron avait dit cela quand il était beaucoup plus jeune, il est très clair que la mort ne peut être prématurée pour lui vingt ans après (Cic. Phil. 2.119) :

(8) Etenim si abhinc annos prope viginti hoc ipso in templo negavi posse mortem immaturam esse consulari, quanto verius nunc negabo seni !

[Car si, il y a quelque vingt ans, dans ce même temple, j'ai déclaré que la mort ne pouvait être prématurée pour un consulaire, avec combien plus de vérité ne dirai-je pas aujourd'hui qu'elle ne peut l'être pour un vieillard].

Là également, Cicéron essaie de construire un ethos favorable et persuasif. Cette fois-ci, il suggère que la possibilité de la mort ne comporte plus aucune horreur pour lui et que ses discours et ses actes sont cohérents.

\subsection{L'argument par la définition}

Mentionnons rapidement un autre argument, celui qui se fonde sur la définition. Ainsi, l'impossibilité d'un compromis ou d'un renoncement partiel à la liberté est confirmée par Cicéron aussi bien par le caractère indissociable de la paix et de la liberté, que par la définition de la servitude comme le plus grand mal. Ce mal (postremum malorum omnium, un superlatif dramatisant qui donne à l'argument sa dimension émotionnelle) doit absolument être éliminé, même s'il en coûte la vie (Cic. Phil. 2.113) :

(9) Et nomen pacis dulce est et ipsa res salutaris; sed inter pacem et servitutem plurimum interest. Pax est tranquilla libertas, servitus postremum malorum omnium, non modo bello sed morte etiam repellendum

[Le nom seul de paix est plein de charme, et la chose elle-même est essentielle à la vie ; mais, entre la paix et la servitude, grande est la différence : la paix est la liberté dans la tranquillité, la servitude le pire de tous les maux ; il faut, pour s'en délivrer, avoir recours non seulement à la guerre, mais même à la mort].

\subsection{L'argument pragmatique, ou l'argument par la peur}

Une autre émotion forte, la peur, est impliquée dans un "argument pragmatique " utilisé par Cicéron. Ce type d'argument causal supporte ou refuse l'exécution d'une action en raison des effets positifs ou négatifs de cette action (pour la structure de ce schème argumentatif cf. Perelman et Olbrechts-Tyteca $1983: 357$ sv. ; Schellens 1985 : 157, Kienpointner $1992: 340$, Garssen $1997: 21$ sv., Walton et al. $2008: 100$ sv.). Cicéron essaie de montrer les effets catastrophiques de l'ambition d'instaurer la tyrannie pour Marc Antoine lui-même ( = Marc Antoine sera assassiné comme César). Par là-même, il cherche à l'intimider, il s'agit donc aussi d'un argument émotionnel ad metum (ou bien ad baculum ; cf. Walton 2000 ; Cic. Phil. 2.117 - et similairement Cic. Phil. 2.1, 2.116) :

(10) Haec non cogitas, neque intellegis satis esse viris fortibus didicisse quam sit re pulchrum, beneficio gratum, fama gloriosum tyrannum occidere? An, cum illum homines non tulerint, te ferent?

[Ne fais-tu pas ces réflexions? Ne comprends-tu pas qu'il suffit à des hommes de cœur d'avoir appris la beauté de l'exploit, la valeur du bienfait et la gloire du renom que comporte le meurtre d'un tyran? Crois-tu que, si on n'a pas pu le (= César) supporter, on te supportera, toi ?] arguments a minore, par la définition et pragmatiques ne rendent pas la conclusion de 
Cicéron absolument convaincante. Ce ne sont toutefois pas les aspects émotionnels qui sont les plus faibles dans son argumentation. $\mathrm{Au}$ contraire, la défense de son engagement pour la liberté républicaine est rendue plus crédible et respectable par la construction de son ethos et sa conviction étant que son âge, sa réputation et ses antécédents ne lui laissent qu'une seule alternative.

\section{L'argument ad hominem comme argument émotionnel}

41 Les arguments discutés jusque-là n'étaient pas des arguments émotionnels intrinsèques, mais des instances de schèmes argumentatifs formulés stratégiquement par Cicéron d'une manière (très) émotionnelle. Je voudrais terminer cette contribution sur une série d'arguments ad hominem qui, plutôt que de traiter seulement de l'objet central de la discussion, sont dirigés contre la personnalité de l'opposant dans la discussion. Je considère que les ad hominem sont des arguments émotionnels intrinsèques dans la mesure où ils impliquent toujours des évaluations négatives et suscitent des émotions négatives comme la haine ou le dédain. Rappelons que Macagno/Walton (2014: 31$)$ soulignent la connexion très étroite entre les évaluations et les émotions: "the strict relationship between ethics, or rather value judgments, and émotions ».

Il existe un certain consensus sur le fait que Cicéron, en accusant Marc Antoine personnellement dans les Philippiques, lance des attaques émotionnelles totalement exagérées en ce qu'elles ne sont pas justifiées par les faits historiques ${ }^{3}$. Or, ces attaques ne peuvent pas être rejetées ou critiquées comme fallacieuses sur la seule base de leur dimension émotionnelle. Il faut plutôt les évaluer dans leur contexte verbal et situationnel.

Avec ses Philippiques, Cicéron veut montrer que les intentions politiques d'Antoine et sa politique d'alors sont mal intentionnées et destructrices: il prétend conserver les structures de la république romaine pour le moment, mais c'est dans le but de devenir en fin de compte un souverain absolu comme César. Pourtant, la politique du consul Marc Antoine immédiatement après l'assassinat de César est modérée (il a accepté une amnistie des meurtriers de César et a laissé abolir la fonction politique de dictatura, décision appréciée même par Cicéron - cf. Phil. 1.3 ; 2.91) ; en outre, comme consul, il a réussi à devenir le leader des césariens tout en gardant des relations relativement paisibles avec les meurtriers de César (cf. Syme 1952: 105: "Antonius displayed consummate skill as a statesman »). Dans ces circonstances, Cicéron se devait donc de saper la crédibilité d'Antoine.

Or, on ne peut pas critiquer les intentions et la crédibilité d'une personne sans utiliser des arguments ad hominem, dirigés contre le caractère, les facultés mentales et les modes de comportement de l'adversaire. Le problème est donc: Quelles instances des arguments ad hominem utilisés par Cicéron sont acceptables et quelles instances sont fallacieuses? Dans la mesure où notre connaissance de la personnalité d'Antoine est largement influencée par les Philippiques de Cicéron et par la propagande d'Octavien (Pasquali 2009: 10 ; Halfmann 2011 : 184 sv.), il est souvent très difficile de différencier la critique justifiée de la calomnie. On peut toutefois distinguer quelques cas où Cicéron manœuvre stratégiquement d'une manière plutôt acceptable, alors que dans d'autres cas ses arguments ad hominem peuvent vraiment être qualifiés de sophismes. 
Avant de présenter et de discuter quelques exemples, je voudrais distinguer les quatre sous-types d'arguments ad hominem selon Walton (1998) : "Circumstantial ad hominem, Direct ad hominem, Bias ad hominem, Situationally Disqualifying ad hominem » (1998: 261). Les ad hominem circonstanciels sont ceux qui relèvent une incohérence ou une contradiction entre ce qu'on dit et ce qu'on fait. Les arguments " ad hominem directs » (dits aussi « abusifs ») blâment le caractère, la compétence ou la morale de l'adversaire. Les arguments « ad hominem biaisés » critiquent la partialité d'une personne. Enfin, les arguments « ad hominem situationnellement disqualifiants» tentent de montrer qu'une personne se trouve dans une situation dans laquelle elle ne peut émettre de jugement valable (1998: 240).

La grande majorité des arguments ad hominem utilisés par Cicéron font partie du soustype ad hominem direct. Ici encore, on peut distinguer cinq « sous-sous-types » (Walton $1998: 215)$ :

1) Negative Ethotic Ad Hominem Argument from Veracity: $a$ has a bad character for veracity ; 2) Negative Ethotic Ad Hominem Argument from Prudence: $a$ has a bad character for prudent judgment; 3) Negative Ethotic Ad Hominem Argument from Perception : $a$ has a bad character for realistic perception of his situation ; 4) Negative Ethotic Ad Hominem Argument from Cognitive Skills : a has a bad character for logical reasoning; 5) Negative Ethotic Ad Hominem Argument from Morals : a has a bad character for personal moral standards.

Pour évaluer ces arguments, Walton (1998: 250) propose les questions critiques (= critical questions ou $C Q$ ) suivantes :

CQ 1

Is the premise true (or well supported) that $a$ is a person of bad character?

CQ 2

Is the issue of character relevant in the type of dialogue in which the argument was used?

CQ 3

Is the conclusion of the argument that [argument] $\alpha$ should be (absolutely) rejected even if other evidence to support $\alpha$ has been presented, or is the conclusion merely (the relative claim) that $\alpha$ should be assigned a reduced weight of credibility, relative to the total body of evidence available? CQ 3 est affirmative et la réponse à la deuxième partie est négative, l'argument ad hominem peut être classifié comme argument fallacieux.

Dans ce qui suit, je négligerai les passages où Cicéron utilise des insultes peu vérifiables, par exemple, quand il qualifie Marc Antoine de prostituée (scortum, Cic. Phil. 2.44), d'ivrogne et de satyre notoire (Cic. Phil. 2.62-63), ou même de bête énorme et détestable (Cic. Phil. 4.12 et similairement Phil. 6.7). Ces invectives ne constituent pas de vrais arguments (cf. Walton 1998 : 217).

Cicéron présente des arguments ad hominem directs selon chacun de ces sous-soustypes. Je dois me contenter d'en citer et analyser quelques-uns. Le premier exemple est un « Negative Ethotic Ad Hominem Argument from Cognitive Skills» :

(11) Haec tu non propter audaciam dicis tam impudenter, sed quia tantam rerum repugnantiam non videas. Nihil profecto sapis. Quid est enim dementius quam, cum rei publicae perniciosa arma ipse ceperis, obicere alteri salutaria ? (Cic. Phil. 2.19) [Non, ce n'est pas l'audace qui te fait prononcer des paroles si impudentes, mais c'est que tu ne t'aperçois pas de pareilles contradictions. Tu n'as évidemment pas 
de moindre bon sens : y a-t-il plus grande déraison, quand on a pris soi-même les armes pour la ruine de l'Etat, que de reprocher á un autre de les avoir prises pour son salut?]

Avec cet argument, Cicéron réagit au reproche d'Antoine, qui critiquait la décision de Cicéron de mobiliser les troupes pour protéger le sénat, Rome et d'autres villes pendant la conjuration catilinaire en octobre 63 avant J.-C. (Cic. Catil. 1.1, 1.7-8). Cicéron souligne la différence entre la protection militaire du sénat contre la conjuration catilinaire et le fait que Marc Antoine se soit accordé une garde de corps de 6000 soldats en 44 av. J-C. (Cic. Phil. 2.112). Mais il ne se contente pas de critiquer l'intention qu'il prête à Antoine de préparer un coup d'Etat avec ces troupes. Alors que cette suspicion était plutôt plausible - justifiée, par ex., par le fait que Marc Antoine avait offert un diadème royal à César pendant la fête des Lupercales, le 15 février 45 av. J.-C. (Cic. Phil 2.85 ; à noter : les doutes de Halfmann $2011: 61$ ), Cicéron attaque également Antoine en le présentant comme incapable de penser sans tomber dans des contradictions.

Comme une bonne partie du sénat romain ne veut pas commencer une guerre civile contre Antoine (Halfmann 2011: 83), mais souhaite plutôt mener des négociations de paix avec lui, ces attaques personnelles excessives sont nécessaires pour atteindre le but stratégique de Cicéron (cf. aussi Cic. Phil. 2.30) : il veut montrer que Marc Antoine est une personne avec laquelle on ne peut pas conclure un pacte, et que la guerre contre lui est la seule solution possible. La pertinence des arguments ad hominem est donc garantie par le contexte argumentatif (CQ 2).

Pourtant, ce « Negative Ethotic Ad Hominem Argument from Cognitive Skills » n'est pas justifié par les faits historiques, qui montrent que Marc Antoine peut se comporter en politicien habile et prudent, s'il le veut (CQ 1 ; cf. Halfmann 2011 : 66). C'est pourquoi on peut juger que cet argument de Cicéron est un argument fallacieux.

L'exemple suivant est un "Negative Ethotic Ad Hominem Argument from Morals». Toujours dans le but de caractériser Marc Antoine comme une personne irresponsable, Cicéron lui reproche d'avoir été informé de l'attentat contre César. Ce reproche peut revendiquer une forme de plausibilité, mais cette fois encore, Cicéron ne se contente pas d'argumenter contre la légitimité de Marc Antoine à assumer une position procésarienne, il y ajoute un reproche de lâcheté. Selon lui, Antoine n'était pas un homme digne de ce nom (Virum res illa quaerebat), et donc trop lâche pour accomplir l'attentat. Dans cette perspective, la crédibilité d'Antoine est une fois de plus endommagée. Il est décrit comme une personne peu respectable en ce qui concerne la morale, parce qu'il n'est pas loyal à César, auquel Antoine doit tout, et en même temps trop lâche pour participer à l'attentat (Cic. Phil. 2.34) :

(12) Quamquam si interfici Caesarem voluisse crimen est, vide, quaeso, Antoni, quid tibi futurum sit, quem et Narbone hoc consilium cum C. Trebonio cepisse notissimum est et ob eius consili societatem, cum interficeretur Caesar, tum te a Trebonio vidimus sevocari. Ego autem - vide, quam tecum agam non inimice - quod bene cogitasti aliquando, laudo ; quod non indicasti, gratias ago ; quod non fecisti, ignosco. Virum res illa quaerebat

[Pourtant, si c'est un crime que d'avoir voulu la mort de César, considère, Antoine, je te prie, ce qui doit advenir de toi, quand il est de notoriété publique qu'à Narbonne tu en avais formé le dessein avec C. Trebonius, et qu'en raison de cette complicité nous avons vu Trebonius, au moment où l'on tuait César, te tirer à l'écart. Quant à moi (vois combien peu je te traite en ennemi), tu as eu une fois une bonne intention, je t'en félicite ; tu n'as pas révélé le complot, je t’en remercie; tu 
ne l'as pas exécuté, je te le pardonne: c'est un homme que réclamait une telle action]. ne peut vraisemblablement douter du courage d'Antoine, sont il a fait plusieurs fois la preuve au combat. (CQ 1 ; cf. Halfmann $2011: 34$ sv., 47, 160). Et ce n'est pas un hasard si Cicéron néglige les exploits militaires d'Antoine, que soit il ne mentionne pas du tout, ou bien qu'il évoque de façon calomnieuse (Cic. Phil. 2.48, 2.55, 2.59). Le fait que Marc Antoine ait été rappelé par Gaius Trebonius immédiatement avant l'attentat (mentionné par Cicéron comme preuve de l'infidélité d'Antoine) peut être expliqué plus plausiblement comme une manœuvre stratégique des meurtriers de César, qui voulaient ainsi s'assurer que Marc Antoine, combattant éprouvé, ne pourra protéger César (Halfmann 2011: 63). On ne peut donc pas conclure que l'absence de Marc Antoine pendant l'attentat soit un signe de complicité (cf. CQ 3).

(13) Ubi est septiens miliens quod est in tabulis quae sunt ad Opis? Funestae illius quidem pecuniae, sed tamen quae nos, si eis quorum erat non redderetur, a tributis posset vindicare [...] Acta enim Caesaris pacis causa confirmata sunt a senatu : quae quidem Caesar egisset, non ea quae egisse Caesarem dixisset Antonius. Unde ista erumpunt, quo auctore proferuntur? Si sunt falsa, cur probantur? si vera, cur veneunt?

[Où sont les sept cent millions de sesterces, qui sont inscrits sur les registres déposés dans le temple d'Opis ? Argent funeste que celui-là, mais qui cependant, si on ne le rendait pas à ceux à qui il appartient, aurait pu nous affranchir de l'impôt sur la propriété. [...] Il est vrai que les actes de César, dans l'intérêt de la paix, ont été ratifiés par le Sénat, les actes authentiques de César, s'entend, et non ceux qu'Antoine a déclarés tels. D'où ceux-ci sortent-ils subitement? Avec quelle garantie sont-ils produits? S'ils sont faux, pourquoi les approuve-t-on ? S'ils sont authentiques, pourquoi en trafique-t-on ?]

Ici, Cicéron met en doute l'honnêteté d'Antoine, et cette attaque a plus de plausibilité que les autres attaques discutées ci-dessus. Il n'est sans doute pas possible de montrer avec certitude combien d'argent Marc Antoine a réellement détourné quand il s'est emparé du trésor public conservé dans le temple d'Opis (il s'agit de 700 millions de sesterces) et quand il s'est lancé dans un commerce profitable avec des documents dont selon Marc Antoine l'origine est césarienne (Pasquali 2009: 66). Mais il est probable qu'il ait profité de cette opportunité formidable pour se débarrasser de ses dettes et s'enrichir considérablement (CQ 1, CQ 3 ; cf. Halfmann 2011 : 69). L'honnêteté de Marc Antoine est donc au moins plausiblement mise en doute par cette attaque ad hominem, qui donc n'est pas un cas évident de sophisme.

\section{Conclusion}

Dans cette étude, j'ai essayé d'éclairer un texte classique, les Philippiques de Cicéron à l'aide des théories contemporaines (et en particulier celles de Plantin et Walton) qui posent que les arguments émotionnels ne sont pas nécessairement fallacieux. Tout d'abord, il a fallu préciser que le concept de "liberté » discuté par Cicéron est très controversé et que ses différentes versions doivent être jugées dans leur contexte historique. Le pathos invoqué par Cicéron pour justifier son dernier combat rhétorique contre Marc Antoine ne doit donc pas être méprisé en raison du fait que le concept de 
liberté dans la république romaine reste très limité en comparaison avec ce qu'il signifie dans une démocratie moderne. Si son ethos dans les Philippiques est aussi convaincant, c'est qu'il a risqué (et a réellement perdu) la vie dans sa lutte pour la liberté républicaine. Il y a un lien étroit entre la conviction de Cicéron qu'il devait se battre pour la liberté de la république à tout prix, et la fréquence élevée de l'argument d'alternatives (« la liberté ou la mort ») dans les Philippiques.

Dans la mesure où les arguments émotionnels sont généralement des arguments relativement faibles, mais pas nécessairement fallacieux, on ne peut pas se contenter de rejeter en bloc ceux qu'utilise Cicéron dans ses Philippiques. En empruntant à la théorie de l'argumentation contemporaine quelques instruments descriptifs et normatifs tels que les schèmes argumentatifs et les questions critiques, $j$ 'ai montré que seule une analyse contextuelle permet l'évaluation des arguments émotionnels comme relativement plausibles ou comme fallacieux. En outre, cette reconstruction explicite de l'argumentation a permis une évaluation plus précise et systématique de la plausibilité globale des arguments de Cicéron. L'analyse de quelques exemples d'arguments d'alternatives, d'arguments a minore, d'argument pragmatique et d'arguments ad hominem a montré que Cicéron réussit parfois à manœuvrer stratégiquement avec succès, en construisant des arguments émotionnels aussi bien persuasifs que plausibles. En même temps, j'ai essayé de montrer que son argumentation reste fallacieuse dans la majorité des cas spécifiques analysés ci-dessus.

\section{BIBLIOGRAPHY}

Aristote. 2007. Rhétorique. Trad. par Pierre Chiron (Paris : Flammarion)

Aristote. 1967. Topiques I-IV. Éd. et trad. par Jacques Brunschwig (Paris : Les Belles Lettres)

Aristote. 2007. Topiques V-VIII. Éd. et trad. par Jacques Brunschwig (Paris : Les Belles Lettres)

Aristoteles. 2002. Rhetorik. Éd. et trad. par Christof Rapp (Berlin : Akademie-Verlag)

Aristoteles. 2004. Topik. Trad. et comm. par Tim Wagner/Christof Rapp (Stuttgart : Reclam)

Bleicken, Jochen. 1962. « Der Begriff der Freiheit in der letzten Phase der Römischen Republik », Historische Zeitschrift 195.1, 1-20

Cicero. 1976. De oratore/Über den Redner. Éd. et trad. par Harald Merklin (Stuttgart : Reclam)

Cicero. 1987. Der Staat. Éd. et trad. par Karl Büchner (München : Artemis)

Cicero. 1993. Die politischen Reden. Éd. et trad. par Manfred Fuhrmann (München : Artemis)

Cicero. 1997. Die Prozessreden. Éd. et trad. par Manfred Fuhrmann (Zürich : Artemis)

Cicéron. 1959. Discours, 19. Philippiques I à IV. Texte établi et traduit par André Boulanger et Pierre Wuilleumier (Paris : Les Belles Lettres)

Cicéron. 1960. Discours, 20. Philippiques, Và XIV. Texte établi et traduit par Pierre Wuilleumier (Paris : Les Belles Lettres) 
Cicéron. 1950-1957 [1922-1930]. De l'orateur. 3 vol. Éd. et trad. par Edmond Courbeau \& Henri Bornecque (Paris : Les Belles Lettres)

Cicéron. 1980. La République. 2 vol. Éd. et trad. par Esther Bréguet (Paris : Les Belles Lettres)

Copi, Irving M. 1990 [1956]. Introduction to Logic (New York : Macmillan)

Eemeren, Frans H. van. 2010. Strategic Maneuvering in Argumentative Discourse (Amsterdam : Benjamins)

Fantham, Elaine. 2005. « Liberty and the People in Republican Rome », Transactions of the American Philological Association 135.2, 209-229

Fuhrmann, Manfred. 1992. Cicero und die römische Republik (München : Piper)

Garssen, Bart. 1997. Argumentatieschema's in pragma-dialectisch perspectief (Amsterdam : IFOTT)

Gilbert, Michael A. 2001. «Emotional Messages », Argumentation 15.3, 239-249

Habermas, Jürgen. 1988. Theorie des kommunikativen Handelns (Frankfurt/M. : Suhrkamp)

Halfmann, Helmut. 2011. Marcus Antonius (Darmstadt : Wissenschaftliche Buchgesellschaft)

Hamblin, Charles L. 1970. Fallacies (London : Methuen)

Hegel, Georg Wilhelm Friedrich. 1970 [1821] : Grundlinien der Philosophie des Rechts (Frankfurt/M. : Suhrkamp)

Kant, Immanuel. 2012 [1788]. Kritik der praktischen Vernunft (Stuttgart : Reclam)

Kienpointner, Manfred. 1992a. Alltagslogik (Stuttgart : Frommann-Holzboog)

Kienpointner, Manfred. 1992b. « How to Classify Arguments », Frans H. Van Eemeren, Rob Grootendorst, J. Anthony Blair \& Charles A. Willard (éds.). Argumentation Illuminated (Amsterdam : Sicsat) 178-188

Kienpointner, Manfred 1993. « The Empirical Relevance of Ch. Perelman's New Rhetoric », Argumentation $7: 4,419-437$

Kienpointner, Manfred 2005. « Racist Manipulation within Austrian, German, Dutch, French and Italian Right-Wing Populism », de Saussure, Louis \& Peter Schulz (éds). Manipulation and Ideologies in the Twentieth Century (Amsterdam : Benjamins), 213-235

Kienpointner, Manfred. 2006. «How to Present Fallacious Messages Persuasively. The Case of the 'Nigeria Spam Letters' « . Houtlosser, Peter \& M.Agnès van Rees (éds.). Considering PragmaDialectics (Mahwah, N.J. : Erlbaum) 161-173

Kienpointner, Manfred. 2013. «Strategic Maneuvering in the Political Rhetoric of Barack Obama ", Journal of Language and Politics 12.3, 357-377

Kienpointner, Manfred. 2014. « Freiheit oder Tod. Zu einem Leitmotiv politischer Rhetorik innerhalb und außerhalb Europas », Ueding, Gert \& Gregor Kalivoda (éds.). Wege moderner Rhetorikforschung (Berlin : de Gruyter), 595-615

Kienpointner, Manfred \& Anna Orlandini. 2005. « La doxa de la justice à travers les langues et les époques ", Revue Internationale des droits de l'antiquité 52, 181-206

Lacey, Walter K. 1986. Cicero : Second Philippic Oration (Warminster, Wilts. : Bolchazy-Carducci) Locke, John. 1824 [1690]. An Essay Concerning Human Understanding. John Locke. The Works in Nine Volumes. vol. 2 (London : Baldwin) 
Macagno, Fabrizio \& Douglas N. Walton. 2014. Emotive Language in Argumentation (Cambridge : Cambridge Univ. Press)

McDermott, William C. 1970. «In Ligarianam », Transactions of the American Philological Association 101, 317-347

Micheli, Raphaël. 2008. «L'analyse argumentative en diachronie : le pathos dans les débats parlementaires sur l'abolition de la peine de mort ", Argumentation et Analyse de Discours 1, 1-26

Micheli, Raphaël. 2010a. L'émotion argumentée (Paris : Cerf)

Micheli, Raphaël. 2010b. " Emotions as Objects of Argumentative Constructions », Argumentation 24.1, 1-17

Micheli, Raphaël. 2014. Les émotions dans les discours (Louvain-la-Neuve : De Boeck)

Mill, John St. 1946 [1859]. On Liberty and Considerations on Representative Government (Oxford : Blackwell)

Narducci, Emanuele. 2009. Cicerone. La parola e la politica (Bari : Laterza)

Pasquali, Johannes. 2009. Marcus Antonius (Bochum : Projekt-Verlag)

Perelman, Chaim \& Lucie Olbrechts-Tyteca. 1983 [1958]. Traité de l'argumentation (Bruxelles : Editions de l'Université de Bruxelles)

Plantin, Christian. 1998. « Les raisons des émotions », Marina Bondi (éd.). Forms of Argumentative Discourse (Bologna : Clueb), 3-50

Plantin, Christian. 2002. "Analyse et critique du discours argumentatif », Koren, Roselyne \& Ruth Amossy (éds.). Après Perelman: Quelles politiques pour les nouvelles rhétoriques? (Paris :

L'Harmattan), 229-263

Plantin, Christian. 2005. L'argumentation (Paris : Presses Universitaires de France).

Plantin, Christian. 2007. « Moving Demonstrations », van Eemeren, Frans H. van, J. Anthony Blair, Charles A. Willard \& Bart Garssen (éds.). Proceedings of the Sixth Conference of the International Society for the Study of Argumentation (Amsterdam : SicSat), 1065-1071

Rubinelli, Sara. 2010. Ars Topica (Dordrecht : Springer)

Schellens, Peter J. 1985. Redelijke argumenten (Utrecht : ICG Printing)

Schmidt, Manfred G. 2010. Demokratietheorien (Wiesbaden : VS-Verlag)

Solomon, Robert C. 1993. The Passions. Emotions and the Meaning of Life (Indianapolis : Hackett)

Stroh, Wilfried. 2008. Cicero. Redner, Staatsmann, Philosoph (München : Beck)

Syme, Ronald. 1952. The Roman Revolution (Oxford : Clarendon).

Tahin, Gábor (2014) : Heuristic Strategies in the Speeches of Cicero. Dordrecht : Springer.

Walton, Douglas N. 1992. The Place of Emotion in Argument (University Park : Pennsylvania State Univ. Press)

Walton, Douglas N. 1996. Argumentation Schemes for Presumptive Reasoning (Mahwah, N.J. : Erlbaum) Walton, Douglas N. 1997. Appeal to Pity (Albany : State Univ. of New York Press)

Walton, Douglas N. 1998. Ad Hominem Arguments (Tuscaloosa : Univ. of Alabama Press)

Walton, Douglas N. 1999. Appeal to Popular Opinion (University Park : Pennsylvania Sate Univ. Press) 
Walton, Douglas N. 2000. Scare Tactics. (Dordrecht : Kluwer)

Walton, Douglas N., Chris Reed \& Fabrizio Macagno. 2008. Argumentation Schemes (Cambridge : Cambridge Univ. Press).

Wirszubski, Chaim. 1967. Libertas als politische Idee im Rom der späten Republik und des frühen Prinzipats (Darmstadt : Wiss. Buchgesellschaft)

\section{NOTES}

1. Sur les aspects raisonnables du discours émotionnel cf. Macagno et Walton $2014: 63 \mathrm{sv}$.

2. T outes les traductions françaises sont citées d'après Boulanger, Wuilleumier et Bréguet (Cicero 1959, 1960, 1980). Sur la notion de "justice» dans le discours latin cf. Kienpointner et Orlandini 2005.

3. Cf. Syme 1952: 104; Fuhrmann 1992: 254; Stroh 2008: 109; Narducci 2009: 418 ; Pasquali 2009 : 89 sv. ; Halfmann $2011: 10$.

\section{ABSTRACTS}

This article tries to show that certain trends in recent contributions to argumentation theory (as manifested especially in the work of Christian Plantin and Douglas Walton) are right in assuming that emotional arguments are not necessarily fallacious. Therefore, it is better to treat emotional arguments as relatively weak arguments, which nevertheless have some plausibility. The central goal of this contribution is a case study: The often very emotional argumentation in the Philippics of Cicero is reconstructed explicitly with the help of the underlying argument schemes, and it is evaluated afterwards with a set of critical questions. This analysis shows that it is necessary to judge the plausibility of emotional arguments within their verbal and historical context, according to well-established criteria. Moreover, it shows that Cicero sometimes manages to construct emotional arguments which are not only persuasive but also plausible. I have also tried to show that his emotional argumentation is fallacious in the majority of the specific cases.

Le présent article se fonde sur certaines contributions de la théorie contemporaine de l'argumentation (et en particulier sur les travaux de Christian Plantin et de Douglas Walton) pour analyser les Philippiques de Cicéron. Ces théories montrent que les arguments émotionnels ne sont pas nécessairement fallacieux : il convient plutôt de les considérer comme relativement faibles mais en même temps, jusqu'à un certain degré, comme plausibles. Dans cette perspective, cette contribution reconstruit l'argumentation souvent très émotionnelle des Philippiques de Cicéron en en dégageant les schèmes argumentatifs sous-jacents, puis tente de l'évaluer à l'aide d'un ensemble de questions critiques. L'analyse confirme qu'il faut juger de la plausibilité des arguments émotionnels dans leur contexte verbal et historique, selon des critères bien établis. Elle montre que Cicéron réussit parfois à construire des arguments émotionnels non seulement persuasifs mais aussi plausibles, même si dans la majorité des cas, son argumentation émotionnelle apparaît comme fallacieuse. 
INDEX

Mots-clés: arguments émotionnels, Cicéron, liberté, questions critiques, schèmes argumentatifs Keywords: argumentation schemes, Cicero, critical questions, emotional arguments, freedom

\section{AUTHOR}

\section{MANFRED KIENPOINTNER}

Université d'Innsbruck 